\title{
A Note on the Extension of Analytic Functions off Real Analytic Subsets
}

\author{
G. NARDELLI and A. TANCREDI
}

ABSTRACT. Let $X$ be a closed analytic subset of an open subset $\Omega$ of $\mathbb{R}^{\mathrm{n}}$. We look at the problem of extending analytic functions from $X$ to $\Omega$.

\section{INTRODUCTION}

Let $X$ be a closed real analytic subset of an open subset $\Omega \subset \mathbb{R}^{\mathrm{n}}$. A real analytic function on $X$ is a function that is locally restriction of real analytic functions on open subsets of $\Omega$. It is well known (cfr. [3]) that every analytic function on $X$ extends to $\Omega$ when $X$ is coherent.

A bigger class of analytic subsets, the $\mathbb{C}$-analytic subsets (cfr. [2], [3]), i.e. the subsets that have global equations in $\Omega$, are characterized by a weaker extension property: every analytic function on $X$ that extends to a neighborhood of $X$ in $\Omega$ extends to the whole $\Omega$. Moreover on every analytic subset without global equations in $\Omega$ there exist functions that

1991 Mathematics Subject Classification: $32 \mathrm{C05}$

Servicio publicaciones Univ. Complutense. Madrid, 1996.

Partially supported by M.V.R.S.T. and C.N.R. 
do not extend to $\Omega$. We prove here that this situation arises even if $X$ is $\mathbb{C}$-analytic, but not purely dimensional of dimension bigger than one.

In [5] it was pointed out that a $\mathbb{C}$-analytic subset $X \subset \mathbb{R}^{\mathbf{n}}$ may be isomorphic to an analytic subset $Y \subset \mathbb{R}^{m}$ that is not $\mathbb{C}$-analytic using a $C^{\infty}$ map inducing an analytic isomorphism $\phi: X \rightarrow Y$. We prove here that such a situation depends on the fact that $\phi$ or $\phi^{-1}$ does not extend analytically and we show by examples that the isomorphism $\phi$ may be simply restriction of rational maps.

Using the results of [6] it is not difficult to consider also the case when $\Omega$ is a general coherent analytic space.

\section{PRELIMINARY REMARKS AND DEFINITIONS}

We recall some definitions and results that we use in the following. We denote by $k$ the field of real numbers $\mathbb{R}$ or the field of complex numbers $\mathbb{C}$.

Let $X$ be a closed analytic subset of an open subset $\Omega \subset k^{n}$. We denote by $\mathcal{O}_{\Omega}$ the sheaf of $k$-valued analytic functions on $\Omega$. An analytic function on $X$ is a function that is locally restriction of analytic functions on open subsets of $\Omega$, i.e. a section of the sheaf $\mathcal{O}_{X}=\left.\left(\mathcal{O}_{\Omega} / \mathcal{J}_{X}\right)\right|_{X}$, where $\mathcal{J}_{X}$ is the sheaf of ideals on $\Omega$ defined by

$$
\mathcal{J}_{X}(V)=\left\{g \in \mathcal{O}_{\Omega}(V) \mid g_{\mid V \cap X}=0\right\}
$$

for every open subset $V$ of $\Omega$. Of course we speak of real analytic functions (subsets) for $k=\mathbb{R}$ and of complex analytic functions (subsets) for $k=\mathbb{C}$. By morphisms of analytic subsets we intend morphisms for the above structures of ringed spaces.

It is well known that for $k=\mathbb{R}$ the sheaf of ideals $\mathcal{J}_{X}$ may not be a coherent $\mathcal{O}_{\Omega}$-module. A real analytic subset such that $\mathcal{J}_{X}$ is coherent is said to be a coherent analytic subset. It is known that the notion of coherence is invariant by isomorphisms.

We call a ringed space $\left(X, \mathcal{O}_{X}\right)$ a real analytic subspace of $\Omega$ when $X=\operatorname{Supp}\left(\mathcal{O}_{\Omega} / \mathcal{J}\right)$ and $\mathcal{O}_{X}=\left.\left(\mathcal{O}_{\Omega} / \mathcal{J}\right)\right|_{X}$, where $\mathcal{J}$ is a coherent sheaf of ideals of $\mathcal{O}_{\Omega}$. Clearly every coherent analytic subset is an analytic subspace. 
A real analytic subset $X$ is called $\mathbb{C}$-analytic if there exists a complex analytic subset $\tilde{X}$ of an open subset $\tilde{\Omega} \subset \mathbb{C}^{\mathbf{n}}$ such that $\Omega \subset \tilde{\Omega}$ and $\tilde{X} \cap \Omega=X$.

Every coherent subset is $\mathbb{C}$-analytic but there exist $\mathbb{C}$-analytic subsets that are not coherent.

Proposition 1. For an analytic subset $X \subset \Omega$ the following conditions are equivalent:

(i) $X$ is $\mathbb{C}$-analytic; that

(ii) there exists a coherent analytic sheaf of ideals $\mathcal{J}$ of $\mathcal{O}_{\Omega}$ such

$$
X=\operatorname{Supp}\left(\mathcal{O}_{\Omega} / \mathcal{J}\right)
$$

(iii) there exist analytic functions $f_{1}, \ldots, f_{k}$ on $\Omega$ such that

$$
X=\left\{x \in \Omega \mid f_{1}(x)=f_{2}(x)=\cdots=f_{k}(x)=0\right\} .
$$

Proof. See [3].

We refer to the third property of the previous proposition by saying that $X$ has global equations in $\Omega$.

$A \mathbb{C}$-analytic subset is said to be $\mathbb{C}$-irreducible if it is not union of two proper closed $\mathbb{C}$-analytic subsets. 1 is well known that a $\mathbb{C}$-irreducible subset may be a reducible subset as it can be seen in the Examples. Every $\mathbb{C}$-analytic subset has a unique representation as an irredundant union of $\mathbb{C}$-irreducible subsets.

Lemma 1. Let $X$ be a closed real analytic subset of an open subset $\Omega \subset \mathbb{R}^{\mathbf{n}}$. If there exists an open neighborhood $U$ of $X$ in $\Omega$ such that $X$ has global equations in $U$, then $X$ has global equations in $\Omega$.

Proof. By Proposition 1 there exists a coherent sheaf of $\mathcal{O}_{U^{-}}$ modules $\mathcal{F}$ such that $X=\operatorname{Supp} \mathcal{F}$. If $\mathcal{G}$ denotes the trivial extension of $\mathcal{F}$ to $\Omega$ then $\mathcal{G}$ is a coherent sheaf too and $X=\operatorname{Supp} \mathcal{G}$. 
Definition 1. A real analytic subset $X$ is called a non pure dimensional subset at a point a if:

(i) $\operatorname{dim} X_{a}=\operatorname{dim} X$;

(ii) for every neighborhood $U$ of a there exists $x \in U$ such that $\operatorname{dim} X_{x}<\operatorname{dim} X$

(iii) $X_{a}$ is irreducible.

$X$ is called a non pure dimensional subset if it is a non pure dimensional subset at some point.

Lemma 2. Let $X \subset \Omega$ be $a \mathbb{C}$-analytic non pure dimensional subset at a. There exists a $\mathbb{C}$-irreducible subset $T$ of $X$, with $\operatorname{dim} T<\operatorname{dim} X$, that contains $a$ and points $c$ arbitrarily near to a such that $X_{c}=T_{c}$.

Proof. Let $s=\operatorname{dim} X$. By a result of [2] $X$ is union of the set $R_{s}(X)$ of the regular points of $X$ of dimension $s$ and of a closed $\mathbb{C}$-analytic subset $T$ of $X$ such that $\operatorname{dim} T<s$. Clearly $a \in T$ since $a \notin R_{s}(X)$. By the hypothesis there exists at least an irreducible component of the germ $T_{a}$ that is not contained in the closure of $R_{s}(X)$. The conclusion follows by replacing $T$ with one of its $\mathbb{C}$-irreducible components that contains $a$ and taking points $c$ not in the closure of $R_{s}(X)$.

Remark 1. We recall some properties of the smallest complex analytic subset that contains a $\mathbb{C}$-analytic subset (cfr. [2], [7]). Given a real analytic subset $X$ of $\Omega \subset \mathbb{R}^{n}$ for every open subset $\Omega$ of $\mathbb{C}^{\mathrm{n}}$ such that $X \subset \tilde{\Omega}$ let $\tilde{X}_{\tilde{\Omega}}$ be the complex analytic subset defined as the intersection of all closed complex analytic subsets of $\tilde{\Omega}$ that contain $X$. Clearly $\bar{X}_{\bar{\Omega}}$ is the smallest complex analytic subset of $\tilde{\Omega}$ that contains $X$ and $X$ is a $\mathbb{C}$-analytic subset of $\Omega$ if and only if there exists an open set $\tilde{\Omega}$ such that $\tilde{X}_{\tilde{\Omega}} \cap \Omega=X$. In this case if $\tilde{\Omega}$ is a small enough neighborhood of $\Omega$ and $X$ is $\mathbb{C}$-irreducible of dimension $s$, then we have:

(1) $\tilde{X}_{\tilde{\Omega}}$ is irreducible of dimension $s$;

(2) if $\tilde{\Omega}_{1}$ is an open neighborhood of $X$ in $\tilde{\Omega}$ then $\tilde{X}_{\tilde{\Omega}} \cap \Omega_{1}$ contains just one irreducible component $\tilde{Z}$ that contains $X$ and $\tilde{Z}=\tilde{X}_{\Omega_{1}}$; 
(3) there exist open sets $\tilde{\Omega}$ such that $\tilde{X}_{\tilde{\Omega}}$ is invariant with respect to the antiinvolution $\sigma$ of $\mathbb{C}^{\mathrm{n}}$ induced by conjugation;

(4) if $X$ and $\tilde{X}$ are irreducible at a point $x \in X$ of dimension $s$, then $\tilde{X}_{x}$, is the complexified of $X_{x}$. $\bar{\Omega}$.

We will use often the notation $\tilde{X}$ without specifying the open set

\section{EXTENSION PROPERTIES}

Let $X$ be a real analytic subset of an open subset $\Omega \subset \mathbb{R}^{\mathbf{n}}$. We say that the extension property holds for $X$ if every analytic function on $X$ extends to $\Omega$.

We say that the weak extension property holds for $X$ if every analytic function on $X$ that extends to an open neighborhood of $X$ in $\Omega$ extends to $\Omega$.

The Theorem B of H. Cartan (cfr. [3]) implies that the extension property holds for every coherent subset. Even if the following theorem too is a consequence, more or less known, of Cartan's results for non reduced spaces (cfr. [6]), we think that may be interesting to produce a short proof of it.

Theorem 1. Let $X$ be a closed analytic subset of $\Omega \subset \mathbb{R}^{\mathrm{n}}$. The weak extension property holds for $X$ if and only if it has global equations in $\Omega$.

Proof. Let us suppose that $X$ has global equations in $\Omega$; by Proposition 1 there exists a coherent sheaf of ideals $\mathcal{J} \subset \mathcal{J}_{X}$ of $\mathcal{O}_{\Omega}$ such that $X=\operatorname{Supp}\left(\mathcal{O}_{\Omega} / \mathcal{J}\right)$. Let us consider the real analytic subspace $\left(X, \tilde{\mathcal{O}}_{X}\right)$ where $\tilde{\mathcal{O}}_{X}=\left.\left(\mathcal{O}_{\Omega} / \mathcal{J}\right)\right|_{X}$ and let $\eta:\left(X, \tilde{\mathcal{O}}_{X}\right) \rightarrow\left(\Omega, \mathcal{O}_{\Omega}\right)$ be the canonical embedding.

Let $f \in \Gamma\left(X, \mathcal{O}_{X}\right)$ be an analytic function such that there exist an open neighborhood $U$ of $X$ in $\Omega$ and an analytic function $g \in \Gamma\left(U, \mathcal{O}_{\Omega}\right)$ such that $f(x)=g(x)$ for every $x \in X$. The function $g$ induces a section $\eta_{U}(g) \in \Gamma\left(X, \tilde{\mathcal{O}}_{X}\right)$ and, by Cartan's theorem $\mathrm{B}$, there exists $h \in \Gamma\left(\Omega, \mathcal{O}_{\Omega}\right)$ such that $\eta_{\Omega}(h)=\eta_{U}(g)$. Since $\eta$ is a morphism of $\mathbb{R}$ ringed spaces the values of $\eta_{\Omega}(h)$ and $\eta_{U}(g)$ at any point $x \in X$ are $h(x)$ 
and $g(x)$ respectively. We conclude that the analytic function $h$ extends $f$.

Let us suppose now that the weak extension property holds for $X$. For every $a \in \Omega-X$ let us consider the analytic function $f^{a}$ : $\Omega-\{a\} \rightarrow \mathbb{R}$ defined by $f^{a}(x)=\|x-a\|^{-2}$ for every $x \in \Omega, x \neq a$. From the hypothesis there exists an analytic function $g^{a}: \Omega \rightarrow \mathbb{R}$ such that $g^{a}{ }_{X}=f^{a}{ }_{\mid X}$ and then we have

$$
X=\bigcap_{a \notin X}\left\{x \in \Omega \mid g^{a}(x)\|x-a\|^{2}=1\right\} .
$$

The conclusion follows since (cfr. [2]) the intersection of any family of $\mathbb{C}$-analytic subsets is a $\mathbb{C}$-analytic subset.

Corollary 1. Any connected component $Y$ of a $\mathbb{C}$-analytic subset $X$ of $\Omega \subset \mathbb{R}^{\mathrm{n}}$ is $\mathbb{C}$-analytic.

Proof. By [1] $X$ is locally connected and then $Y$ is open and closed in $X$. It follows that any analytic function defined on an open neighborhood of $Y$ in $\Omega$ extends to an open neighborhood of $X$ in $\Omega$. The conclusion follows from the previous theorem.

Corollary 2. If $X$ has not global equations in $\Omega$ the extension property does not hold for $X$.

In the following theorem we prove that the extension property does not hold for non purely dimensional subsets of dimension bigger than one. Of course the condition on the dimension is necessary since all analytic subsets of dimension one are coherent.

Theorem 2. Let $X$ be a $\mathbb{C}$-analytic subset of $\Omega \subset \mathbb{R}^{\mathbf{n}}$ with $\operatorname{dim} X=s \geq 2$. If $X$ is non pure dimensional then there exist analytic functions on $X$ that do not extend to any open neighborhood of $X$ in $\Omega$.

Proof. Let us first assume that $X$ is $\mathbb{C}$-irreducible.

In $\mathrm{X}$ we can choose a point $a$ that verifies the conditions of Definition 1. From Lemma 2 there exists a $\mathbb{C}$-irreducible $\mathbb{C}$-analytic subset $T$, with $\operatorname{dim} T<s, a \in T$, that contains a point $c$ such that $T_{c}=X_{c}$. With the notations of the Remark 1 there exist a Stein open set $\bar{\Omega} \subset \mathbb{C}^{\mathbf{n}}$ such 
that $\Omega=\tilde{\Omega} \cap \mathbb{R}^{\mathrm{n}}$ and analytic subsets $\tilde{X}, \tilde{T}$ in $\tilde{\Omega}$ such that $\tilde{X} \cap \Omega=X$, $\tilde{T} \cap \Omega=T, \operatorname{dim} \tilde{X}=\operatorname{dim} X, \operatorname{dim} \tilde{T}=\operatorname{dim} T, \tilde{X}$ and $\tilde{T}$ irreducible. We can assume $\tilde{\Omega}, \tilde{X}, \tilde{T}$ invariant with respect to the antiinvolution $\sigma$ of $\mathbb{C}^{\mathrm{n}}$ induced by conjugation.

Let us consider the analytic set

$$
\tilde{C}=\{z \in \tilde{\Omega} \mid \tilde{q}(z)=0\}
$$

where

$$
\tilde{q}(z)=\sum_{i=1}^{n}\left(z_{i}-c_{i}\right)^{2} .
$$

Being $\tilde{C} \cap \tilde{X}$ a proper subset of $\tilde{X}$ it is easy to see that the dimension of $\tilde{C} \cap \tilde{X}$ is $s-1$ at every point.

Let us denote by $\tilde{S}^{1}, \ldots, \tilde{S}^{p}$ the irreducible components of $\tilde{C} \cap \tilde{X}$ that contain $c$. For each $j=1, \ldots, p, \sigma\left(\tilde{S}^{j}\right)$ is an analytic irreducible subset of $\tilde{C} \cap \tilde{X}$ of dimension $s-1$. The condition $c \in \sigma\left(\tilde{S}^{j}\right)$ implies that there exists $k(j)$ such that

$$
\sigma\left(\tilde{S}^{j}\right)=\tilde{S}^{k(j)} .
$$

Since $\tilde{S}^{j} \neq \tilde{T}$, for each $j=1, \ldots, p$, there exists

$$
\zeta_{j} \in \tilde{S}^{j}-\tilde{T}
$$

and

$$
\zeta_{j} \notin \tilde{S}^{l} \text { for } l \neq j .
$$

By Cartan's Theorem A there exists a complex analytic function $\hat{h}_{j} \epsilon$ $\mathcal{O}_{\mathrm{a}^{\mathrm{n}}}(\tilde{\Omega})$ such that

$$
\begin{aligned}
& \hat{h}_{j}\left(\zeta_{j}\right) \neq 0, \\
& \hat{h}_{j}(z)=0 \text { for every } z \in \bigcup_{l \neq j} \tilde{S}^{l} \cup \tilde{T} .
\end{aligned}
$$

Let $\tilde{g}_{j}$ be the function 


$$
z \mapsto \frac{1}{2}\left(\hat{h}_{j}(z)+\overline{\hat{h}_{j}(\bar{z})}\right) .
$$

If $k(j)=j$, by multiplying if necessary $\hat{h}_{j}$ by the imaginary unit, we can assume that

$$
\begin{aligned}
& \tilde{g}_{j}\left(\zeta_{j}\right) \neq 0, \\
& \tilde{g}_{j}(z)=0 \text { for every } z \in \bigcup_{l \neq j} \tilde{S}^{l} \cup \tilde{T} .
\end{aligned}
$$

If $k(j) \neq j$, we have

$$
\begin{aligned}
\tilde{g}_{j}(z) & =0 \text { for every } z \in \bigcup_{l \neq j, k(j)} \tilde{S}^{l} \cup \tilde{T}, \\
\tilde{g}_{j}\left(\zeta_{j}\right) & =\frac{1}{2} \hat{h}_{j}\left(\zeta_{j}\right), \\
\tilde{g}_{j}\left(\sigma\left(\zeta_{j}\right)\right) & =\frac{1}{2} \overline{\hat{h}_{j}\left(\zeta_{j}\right)},
\end{aligned}
$$

and then $\tilde{g}_{j}$ is a function not identically zero on $\tilde{S}^{j} \cup \tilde{S}^{k(j)}$ such that $\tilde{g}_{j}(\Omega) \subset \mathbb{R}$. Let us call $j_{1}, \ldots, j_{u}, h_{1}, \ldots, h_{v}, l_{1}, \ldots, l_{v}$, where $u+2 v=p$, the index $\left(j_{i}\right)_{i=1, \ldots, p}$ according to

$$
\sigma\left(\tilde{S}^{j_{i}}\right)=\tilde{S}^{j_{i}} \text { or } \sigma\left(\tilde{S}^{h_{i}}\right)=\tilde{S}^{l_{m}}
$$

and let

$$
\tilde{g}=\left(\sum_{i=1}^{u} \tilde{g}_{j_{i}}+\sum_{t=1}^{v} \tilde{g}_{h_{\imath}}\right)^{2} .
$$

The function so defined is identically zero on $\tilde{T}$, hence on $T$, but it is not identically zero on any irreducible component $\tilde{S}^{j}$ and $\tilde{g}(\Omega) \subset \mathbb{R}$ as it follows easily from (2.2) and (3.2).

Let us now consider the real analytic functions on $\Omega$

$$
g=\left.\tilde{g}\right|_{\Omega} \text { and } q=\left.\tilde{q}\right|_{\Omega}
$$


and let us denote by $\lambda: X \rightarrow \mathbb{R}$ the function defined by

$$
\lambda(x)= \begin{cases}\frac{q(x)}{q(x)+g(x)} & x \neq c \\ 1 & x=c .\end{cases}
$$

In order to prove that $\lambda$ does not extend to any open neighborhood of $X$ in $\Omega$ by Theorem 1 it is enough to prove that it does not extend to $\Omega$. We can suppose that there exists a real analytic function $\mu: \Omega \rightarrow \mathbb{R}$ such that $\mu_{\mid X}=\lambda$. On a suitable open subset $\tilde{\Omega}^{\prime} \subset \tilde{\Omega}$ there exists a complex analytic function $\tilde{\mu}$ such that $\tilde{\mu}_{\mid \Omega}=\mu$. Let $\tilde{X}^{\prime}$ be the irreducible component of $\tilde{\Omega}^{\prime} \cap \tilde{X}$ that contains $X$ and $\tilde{T}^{\prime}$ the irreducible component of $\tilde{\Omega}^{\prime} \cap \tilde{T}$ that contains $T$.

Let

$$
\tilde{P}=\left\{z \in \tilde{X}^{\prime} \mid \tilde{q}(z)+\tilde{g}(z)=0\right\}
$$

and

$$
\tilde{S}=\left\{z \in \tilde{X}^{\prime} \mid \tilde{q}(z)=0\right\}
$$

We observe that

$$
c \in \tilde{P} \cap \tilde{S}
$$

and

$$
\operatorname{dim} \tilde{S}=s-1 .
$$

Moreover $\tilde{P} \subsetneq \tilde{X}^{\prime}$ because $\tilde{q}(a) \neq 0$ while $\tilde{g}(a)=0$; hence $\tilde{X}^{\prime}-\tilde{P} \neq \emptyset$ is an irreducible analytic subset too.

Let $\tilde{\lambda}: \tilde{\Omega}-\tilde{P} \rightarrow \mathbb{C}$ be the complex analytic function defined by

$$
\tilde{\lambda}(z)=\frac{\tilde{q}(z)}{\tilde{q}(z)+\tilde{g}(z)} .
$$

Now we can suppose that arbitrarily near to $a$ there exists a point $x \in X$ with $\operatorname{dim} X_{x}=s$ that is regular for $X$ and $\tilde{X}$. It follows that $\tilde{X}^{\prime}$ is the complexified of $X$ at $x$ and since $\bar{\lambda}$ is defined in a neighborhood of $a$ it results $(\tilde{\lambda}-\tilde{\mu})_{\mid X_{x}}=0$ and so $(\tilde{\lambda}-\tilde{\mu})_{\left.\right|_{X_{x}^{\prime}}}=0$. By the analy tic continuation principle we can conclude 


$$
\bar{\lambda}=\tilde{\mu} \text { over } \bar{X}^{\prime}-\tilde{P} \text {. }
$$

Since $\tilde{g}_{c}$ does not vanish on any $\bar{S}_{c}^{j}, j=1, \ldots, p, \tilde{S} \cap \tilde{P}$ is a non empty proper subset of $\bar{S}$ with

$$
\operatorname{dim}_{c}(\tilde{S} \cap \tilde{P})<s-1 .
$$

Indeed if were $\operatorname{dim}_{c}(\tilde{S} \cap \tilde{P})=s-1$ every irreducible component of $(\tilde{S} \cap \tilde{P})_{c}$ would be an irreducible component of some $\tilde{S}_{c}^{j}$ but then $\tilde{g}$ would be identically zero on $\tilde{S}^{j}$. So the set

$$
\tilde{A}=\tilde{S}-\tilde{S} \cap \tilde{P}
$$

is an open subset of $\tilde{S}$ such that $c \in \overline{\tilde{A}}$. But

$$
\bar{\lambda}(z)=\tilde{\mu}(z)=0 \text { for every } z \in \overline{\tilde{A}}
$$

and so

$$
\tilde{\mu}(c)=0
$$

because $\tilde{\mu}$ is continuous in $c$. This contradicts the assumption

$$
\tilde{\mu}(c)=\mu(c)=1 .
$$

If $X$ is $\mathbb{C}$-reducible there exists a unique $\mathbb{C}$-irreducible component $X^{\prime}$ of $X$ that contains $a$. By the above construction we find an analytic function on $X^{\prime}$ that does not extend to $\Omega$. Since we can assume that $c$ does not belong to any $\mathbb{C}$-irreducible component of $X$ except $X^{\prime}$ the analytic function we found is well defined on the whole $X$ and, of course, does not extend to $\Omega$.

Corollary 3. If $X$ is normal, i.e. if the $\mathbb{R}$-algebra $\mathcal{O}_{X, x}$ is integrally closed in its quotient field for every $x \in X$, then the following conditions are equivalent:

(i) the extension property holds for $X$;

(ii) $X$ is coherent;

(iii) $H^{1}\left(X, \mathcal{J}_{X}\right)=0$. 
Proof. By [6] a normal analytic space is coherent if and only if it is of pure dimension.

\section{GLOBAL EQUATIONS}

Theorem 3. Let $X \subset \mathbb{R}^{\mathrm{n}}, \mathrm{Y} \subset \mathbb{R}^{\mathrm{m}}$ be real analytic subsets, $\phi$ : $X \rightarrow Y$ an analytic isomorphism such that $\phi$ has an analytic extension to $\mathbb{R}^{\mathrm{n}}$ and $\phi^{-1}$ has an analytic extension to $\mathbb{R}^{\mathrm{m}}$. Then $X$ is $\mathbb{C}$-analytic if and only if $Y$ is $\mathbb{C}$-analytic.

Proof. Let us suppose that $\phi$ and $\phi^{-1}$ are induced respectively by analytic maps $\psi: \mathbb{R}^{\mathbf{n}} \rightarrow \mathbb{R}^{\mathbf{m}}$ and $\theta: \mathbb{R}^{\mathbf{m}} \rightarrow \mathbb{R}^{\mathbf{n}}$. Let us denote by $\hat{X}$ and $\hat{Y}$ the smallest $\left(\mathbb{C}\right.$-analytic subsets of $\mathbb{R}^{\mathbf{n}}$ and $\mathbb{R}^{\mathbf{m}}$ respectively that contain $X$ and $Y$. Every analytic function identically zero on $X$ (on $Y$ ) is identically zero on $\hat{X}$ (on $\hat{Y}$ ). It follows that $\left.\theta \psi\right|_{\hat{X}}=\mathrm{id}_{\hat{X}}$ and $\left.\psi \theta\right|_{\hat{Y}}=\mathrm{id}_{\hat{Y}}$, i.e. $\hat{X}$ is isomorphic to $\hat{Y}$. Now $X$ is $\mathbb{C}$-analytic if and only if $X=\hat{X}$ and we have $\theta(\hat{Y})=X=\theta(Y)$ if and only if $\hat{Y}=Y$.

Examples. We will produce examples of analytic subsets $X$ and $Y$ that are isomorphic, but only one of them is $\mathbb{C}$-analytic.

Let

$$
X=\left\{x \in \mathbb{R}^{3} \mid\left(1-\mathrm{x}_{3}\right) \mathrm{x}_{1}^{2}+\mathrm{x}_{2}^{2}=0\right\}
$$

be the classical Whitney umbrella and

$$
\begin{aligned}
& Z=\left\{x \in \mathbb{R}^{3} \mid\left(1-\mathrm{x}_{3}\right) \mathrm{x}_{3}^{2} \mathrm{x}_{1}^{2}+\mathrm{x}_{2}^{2}=0\right\}, \\
& Y=\left\{x \in Z \mid x_{3} \neq 0\right\} \cup\{0,0,0\}, \\
& L=\left\{x \in \mathbb{R}^{3} \mid \mathrm{x}_{2}=\mathrm{x}_{3}=0\right\} .
\end{aligned}
$$

It is easy to see that $Y$ is a closed analytic subset of $\mathbb{R}^{3}$ and $Z=$ $Y \cup L$

We want to show that $Y$ is not $\mathbb{C}$-analytic: by Lemma 1 , it is enough to show that every analytic function $f: \mathbb{R}^{3} \rightarrow \mathbb{R}$ that vanishes on $Y$ vanishes on $L$ too. There exist an open neighborhood $\tilde{\Omega}$ of $\mathbb{R}^{3}$ in $\mathbb{C}^{3}$ 
and a complex analytic function $\tilde{f}: \tilde{\Omega} \rightarrow \mathbb{C}$ such that $\left.\tilde{f}\right|_{\mathbf{R}^{3}}=f$. Let us consider now the complex analytic subset

$$
\tilde{Z}=\left\{z \in \mathbb{C}^{3} \mid\left(1-\mathrm{z}_{3}\right) \mathrm{z}_{3}^{2} \mathrm{z}_{1}^{2}+\mathrm{z}_{2}^{2}=0\right\},
$$

and the path $\gamma:[0,1] \rightarrow \mathbb{C}^{3}$ defined by

$$
\gamma(t)=\left(\epsilon(1-t), \quad i \epsilon t(1-t)^{\frac{3}{2}}, t\right)
$$

where $\epsilon$ is a positive small enough real number. It is easy to see that $\gamma(t) \in \tilde{Z} \cap \tilde{\Omega}$ for every $t \in[0,1]$ and for $t \neq 0,1$ it is a regular point of $\bar{Z}$. The complex analytic subset $\bar{Z}$ is the complexified of $Z$ at the point $\gamma(1)$ and since the real analytic function $f$ vanishes on a neighborhood of $\gamma(1)$ in $Z$ the complex analytic function $\vec{f}$ vanishes on a neighborhood of $\gamma(1)$ in $\tilde{Z}$. It follows that $\tilde{f}(\gamma(0))=f(\gamma(0))=0$, but $\gamma(0) \in L-Y$.

We observe that $Z$ is a $\mathbb{C}$-analytic $\mathbb{C}$-irreducible subset, but it is union of two closed proper irreducible subsets: $Y$ that is not $\mathbb{C}$-analytic and $L$ that is trivially coherent.

Let us denote by $\phi: X \rightarrow \mathbb{R}^{3}$ the analytic map defined by

$$
\phi(x)= \begin{cases}\left(\frac{x_{1}}{x_{3}}, x_{2}, x_{3}\right) & x_{3} \neq 0 \\ (0,0,0) & x_{3}=0\end{cases}
$$

and $\psi: Y \rightarrow X$ the analytic map $\psi: x \mapsto\left(x_{1} x_{3}, x_{2}, x_{3}\right)$. It is straightforward to see that $\psi \phi=\mathrm{id}_{X}$ and $\phi \psi=\mathrm{id}_{Y}$.

A similar example may be produced even if $\phi$ extends to $\mathbb{R}^{3}$ by considering the analytic map on $X \phi: x \mapsto\left(x_{1}, x_{2} x_{3}, x_{3}\right)$ and the analytic map on $Y$ defined by

$$
\psi(x)= \begin{cases}\left(x_{1}, \frac{x_{2}}{x_{3}}, x_{3}\right) & x_{3} \neq 0 \\ (0,0,0) & x_{3}=0\end{cases}
$$

\section{References}

[1] Bruhat, F. and Cartan,R., Sur la structure de sous-ensembles analytiques réels, C.R. Acad. Sci. Paris 244 (1957), 988-990.

[2] Bruhat, F. and Whitney, H., Quelques propriétés fondamentales des ensembles analytiques réels, Comm. Math. Helv. 33 (1959), 132-160. 
[3] Cartan, H.. Variétés analytiques réelles et variétés analytiques complexes, Bull. Soc. Math. France 85 (1957), 77-99.

[4] Narashiman, R., Introduction to the theory of analytic spaces, Lecture Notes in Mathematics 25, Springer, Berlin, Heidelberg, New York, 1966.

[5] Tancredi, A., Un'osservazione sui sottoinsiemi $\mathbb{C}$-analitici, Ann. Univ. Ferrara 29 (1983), 221-224.

[6] Tognoli, A., Proprietà globali degli spazi analitici reali, Ann. Mat. Pura e Appl. 75 (1967), 143-218.

[7] Tognoli, A., Pathology and imbedding problems for real analytic spaces, Singularities of analytic spaces, Cremonese, Roma, 1975, pp. 162180 .

Dipartimento di Matematica

Università di Perugia

Via Vanvitelli, 1 I-06123 Perugia,

ITALY

E-mail address: nardelli@gauss.dipmat.unipg.it
Dipartimento di Matematica

Università di Perugia

Via Vanvitelli, 1 I-06123 Perugia

ITALY

E-mail address: altan Qunipg.it

Recibido: 17 de Noviembre, 1994

Revisado: 19 de Enero, 1995 